【研究简报】

\title{
单个准分子激光脉冲和位相光栅 模板制作表面周期结构
}

\author{
骆桂蓬(1)吴海明电王牧 ${ }^{(1)}$ 祝世宁 (1) 陆延青D

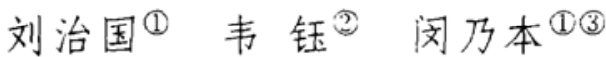

(1)南京大学固体潄结构物理国家重点实验室,南京 210093; (3) 东南大学分子与生物分子电子学实验室, 南京 210096; (3) CCAST World Laboratory, 北京 100080)

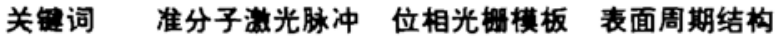

近年来, 准分子激光器 (excimer laser) 以其高能量的脉冲式紫外 (193 nm 或 $248 \mathrm{~nm}$ ) 激光 输出特征而被人们用于部分薄膜材料的加工和制备 ${ }^{[1,2]}$. 其原理 是用聚焦后的准分子激光 脉冲对靶材进行轰击, 一定条件下将轰击出来的靶材颗粒均匀沉积在基片上便可以生长出薄 膜结构, 这种方法又称为 PLD (Pulse laser deposition) 技术. 利用准分子激光器和石英位相光 棚模板, 人们已经在光敏光纤和光敏光波导中写入了性能良好的 Bragg 折射率光栈 ${ }^{[3,4]}$. 由 于 $\mathrm{LiNbO}_{3}(\mathrm{LN})$ 和 $\mathrm{LiTaO}_{3}(\mathrm{LT})$ 这两种重要的光学晶体均对准分子激光波长 $(248 \mathrm{~nm})$ 产生强 烈吸收, 所以当它们被该激光照射时, 激光能量将被晶体吸收并产生热量. 如果激光的能量密 度很高, 就会将晶体表层直接气化后剥离样品; 如果激光能量适中, 晶体表层迅速融化后并不 脱离基片, 而是冷却后在表面重新凝固. 为此, 我们设想如果将两束相干的激光形成的干涉条 纹照射到晶体表面,那么在合适的激光能量密度下可以使被激光周期状融化的晶体表层再凝 固时发生形状起伏, 从而在晶体表面刻上几何光㮽条纹. 本文首次提出利用准分子激光脉冲, 可以在一些重要的光学晶体表面制作周期结构。实验表明, 该方法可以在 LN 和 LT 晶体表 面获得良好的位相光桶。由于该方法工艺简单, 制作方便, 非常适合工业化生产, 因此具有十 分重要的研究和应用价值.

10 Shirai $\mathrm{Y}$, Schaefer H E, Seeger A. Study of thermal equilibrium vacancies in pure iron by positron lifetime spectroscopy, In: Dorikens Vanpraet L, Dorikens M, Segers D, eds. Positron Annihilation. New Jersey, Hong Kong, Singapore: ICPA-8. 1988. $419 \sim 421$

11 Brandt W, Dupasquier A. Positron Solid-State Physics, Amsterdam, New York, Oxford: . Vorth Holland, 1983.200

12 Lock D G, West R.N. Positron annihilation in disordered binary alloys. J Phys F: Met Phys, 1974, 4(12): 2 179 - 2188

13 West R N. Positron studies of condensed matter. Adv Phys, 1973, 22: 263 383

14 Stott M J, Kubica. New approach to the positron distribution in metals and alloys. Phys Rev B, 1975, 11(1): 1 10

15 Hong T, Freeman A J. Effect of stoichiometry on the structural properties and the electronic structure of intermetallics: antiphase boundary energies in FeAl and NiAl. J Mater Res, 1992, 7(1): 68 79

16 Lin W, Xu Jian-hua, Freeman A J. Cohesive properties, electronic structure and bonding characteristics of Ru. $\mathrm{d}$-a coparison to NiAl. J Mater Res, 1992, 7(3): 592 604 


\section{1 实验装置与结果}

本文所采用的实验系统如图 1 所 示. 从 $\mathrm{KrF}$ 准分子激光器 (Lambda Physik Model LPX205i) 发出的波长为 $248 \mathrm{~nm}$ 的紫外激光束, 其空间分布为矩 形 $(7 \mathrm{~mm} \times 22 \mathrm{~mm})$, 脉冲宽度为 $30 \mathrm{~ns}$. 经过柱面透镜和反射镜后入射到光栅模 板上，柱面透镜由纯石英玻璃制成，焦 距为 $180 \mathrm{~mm}$; 反射镜由光学玻璃表面

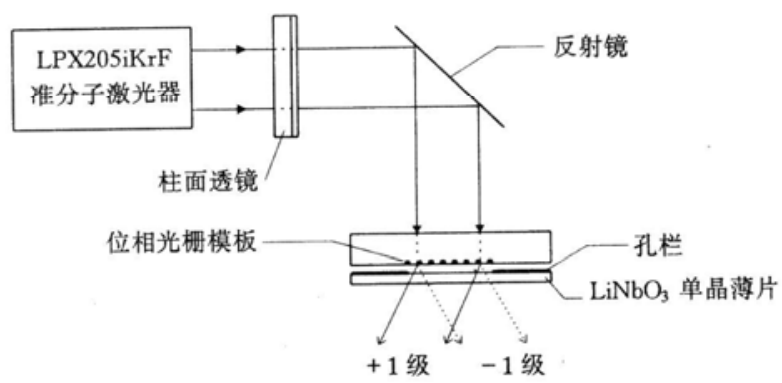

图 1 准分子激光刻蚀晶体光棚实验系统 镀一层铝制成, 对 $248 \mathrm{~nm}$ 的激光反射率大于 $90 \%$ 。柱面透镜的作用是将矩形激光束压缩为 近似正方形 $(7 \mathrm{~mm} \times 7 \mathrm{~mm}$ ) 光束, 从而提高激光的能量密度. 尺寸为 $4.5 \mathrm{~mm} \times 4.5 \mathrm{~mm}$ 的孔 栏(厚度为 $500 \mu \mathrm{m}$ ), 放置在样品和模板之间, 每次照射仅用一个激光脉冲.

位相光栅模板 ( $5 \mathrm{~mm} \times 5 \mathrm{~mm}$ ) 采用纯石英玻璃(厚 $2 \mathrm{~mm}$, 对紫外波长透过率大于 $95 \%$ ) 制 成. 光棚由等间距的方形刻槽组成, 刻槽深度应设计为对 $248 \mathrm{~nm}$ 的准分子激光发生 0 级掩 模, 即当激光垂直入射到光棚上时, 相邻的两束 0 级光相互抵消, 使主要的能量分布在 \pm 1 级 上, 从而实现周期为 $d / 2$ 的干涉条纹. 此时 $h=\lambda / 2(n-1)$, 代入参数 $\lambda=248 \mathrm{~nm}, n=1.47$, 得到 $h \approx 270 \mathrm{~nm}$. 由于刻蚀和腐蚀技术的限制, 模板的条纹刻槽往往达不到理想的等间距方 形, 加上腐蚀深度的误差, 使得模板对 $248 \mathrm{~nm}$ 准分子激光的 0 级衍射仍然存在. 尽管如此, 只 要衍射能量主要分布在 \pm 1 级上, 便可以实现较强的干涉条纹. 经测试, 所用模板在激光垂直 入射时 0 级约占 $16 \%, \pm 1$ 级约各占 $32 \%$, 其他级次约占 $20 \%$. 模板实际深度为 $(260 \pm 10) \mathrm{nm}$.

LN 和 LT 单晶薄片样品尺寸为 $10 \mathrm{~mm} \times 40 \mathrm{~mm}$, 厚 $1 \mathrm{~mm}$, 表面的晶格取向为 $(006)$, 经光 学抛光后放在实验系统中的指定位置, 用单个准分子激光脉冲在不同激光能量下进行刻蚀实 验. 刻蚀后的光栅分别在光学显微镜和原子力显微镜下进行形貌观察和记录.
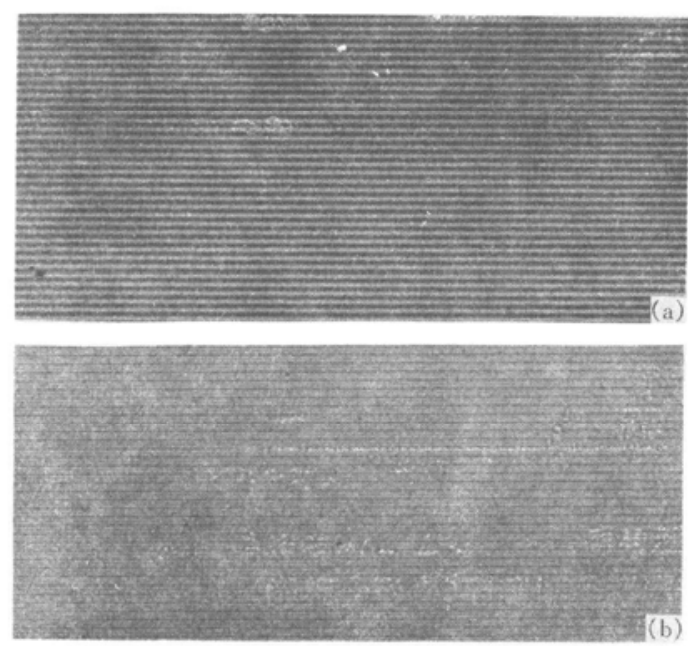

图 $2 \mathrm{LN}$ 光棚的光学显微照片 (a)中间区域; (b)边界区域

图 2 给出了在 $250 \mathrm{~mJ}$ 的单个激光脉冲照 射后 LN 晶体表面的光学显微镜照片, 可以看 出在激光的照射区域形成了表面周期结构。值 得注意的是, 在照射区域的不同位置观察到两 种周期的光栅结构, 一种是周期尺寸约为 2 $\mu \mathrm{m}$, 且深度均匀的光栅, 它与模板的周期相同, 位于照射区域中部(图 2(a)); 另一种是周期为 $1 \mu \mathrm{m}$ (为模板周期的一半) 的光棚, 多位于照射 区边界, 但刻槽的深度周期变化, 即两个深刻槽 之间存在一个浅刻槽(图 2(b))．尽管形成两 种周期光棚的过程并不十分清楚, 但从实际的 实验条件及参数可以作出如下解释.

上面已经讨论过, 在理想条件下, 即垂直 入射的激光束透过位相光桷模板后只有 \pm 1 级衍射光时, 则在模板下表面的干涉区域将 
形成周期为模板周期一半的干涉条纹. 但是, 如果 0 级光不能被消除, 则它将与 \pm 1 分别相 干, 从而形成与模板周期相同的干涉条纹. 本实验模板是针对垂直入射条件下 0 级被抵消时 设计的, 而当激光束按一定角度入射到模板上时, 0 级光强将增大, 并与 \pm 1 级光干涉产生与模 板周期相同的干涉条纹. 在实际实验中, 激光束并不能被调整到理想的垂直条件, 加上激光束 本身的发散角, 所以, 入射到样品上的激光束虽然在中心区域近似垂直, 但在边界却有一定的 入射角. 根据公式计算, 在本实验所用的光栅模板下, 当入射角 $\theta=\operatorname{arc} \sin (\lambda / 2 \Lambda)=3.95^{\circ}$ 时, 0 级光便可以和 \pm 1 级发生干涉, 形成与模板相同周期的光棚条纹, 因此整个照射区域便可能 形成两种不同周期的光梛条纹. 图 3 给出原子力显微镜 (AFM)下, $L N$ 晶体光棚的表面形貌.

在 AFM 图象中, $L N$ 晶体光棚的表面形貌十分清晰 (见图 3). 从图 3(a) 中可以看出, 光 棚 (中间区域) 具有很好的周期性和均匀性; 图 3(b) 显示其表面为波纹状的起伏, 深度约为

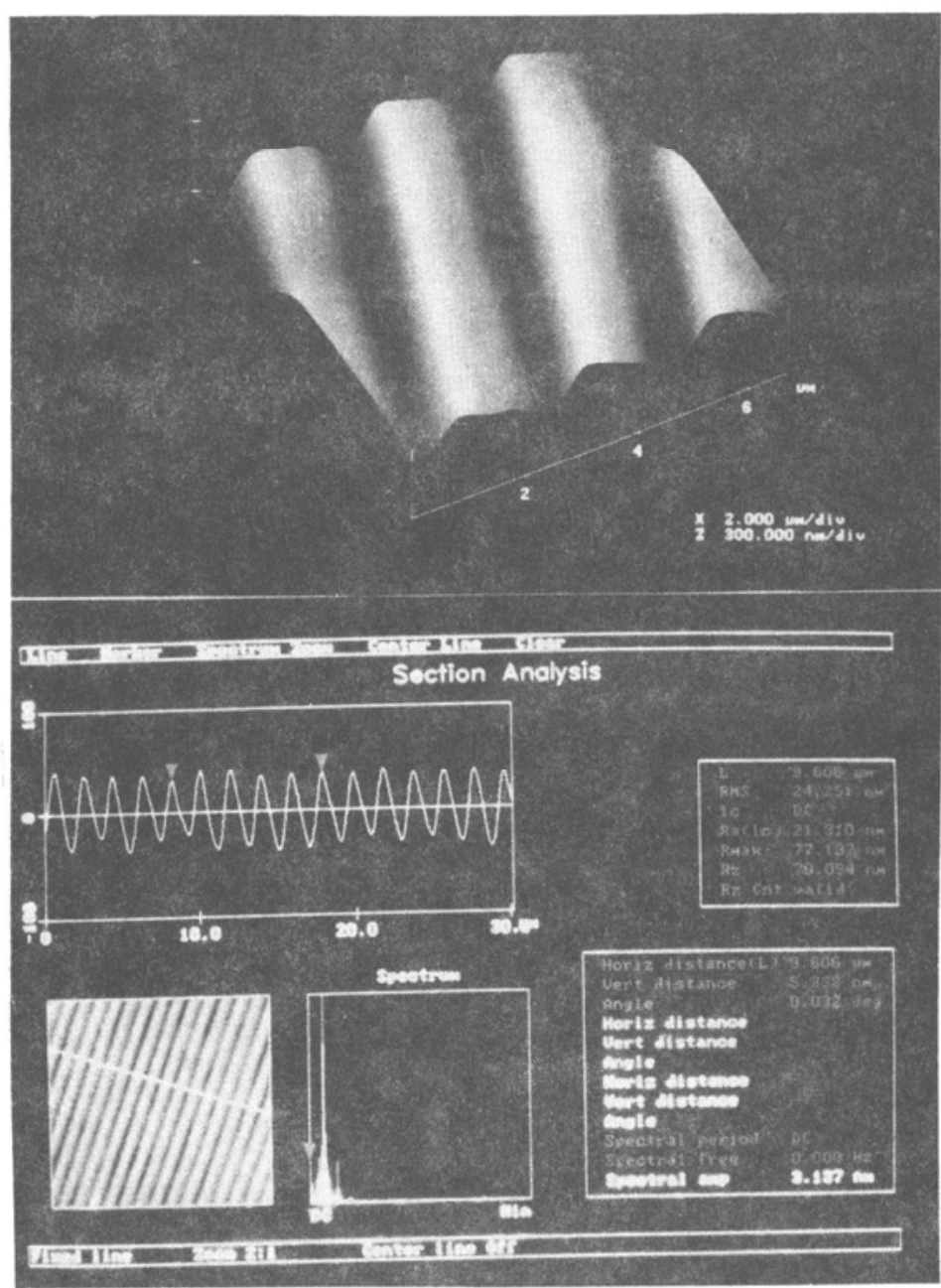

图 $3 \mathrm{LN}$ 光相的 $\mathrm{AFM}$ 照片 (a)三维形貌; (b) 剖面结构 
$110 \mathrm{~nm}$, 且光栅刻槽内部具有纳米量级的表面光洁度. LT 光栅的刻蚀结果与 LN 相似, 只是 由于它们的熔点不同, 刻蚀同样的光栅深度需要不同的激光能量密度. 由于刻蚀结果与 LN 光栅类似, 就不详细讨论了.

\section{2 光栅刻蚀机理的分析与讨论}

为了清楚地了解光栅的形成过程, 本节利用原子力显微镜, 通过观察光棚边界的起伏变 化, 判断光栅形成的物理过程. 图 4 给出了 LN 光栅的边界处 AFM 照片, 从光栅的横断面 $\mathrm{AFM}$ 照片 (图4b) 可以看出, 光柝波纹的峰值高出了原来的晶体表面 (图中的水平线), 而且高

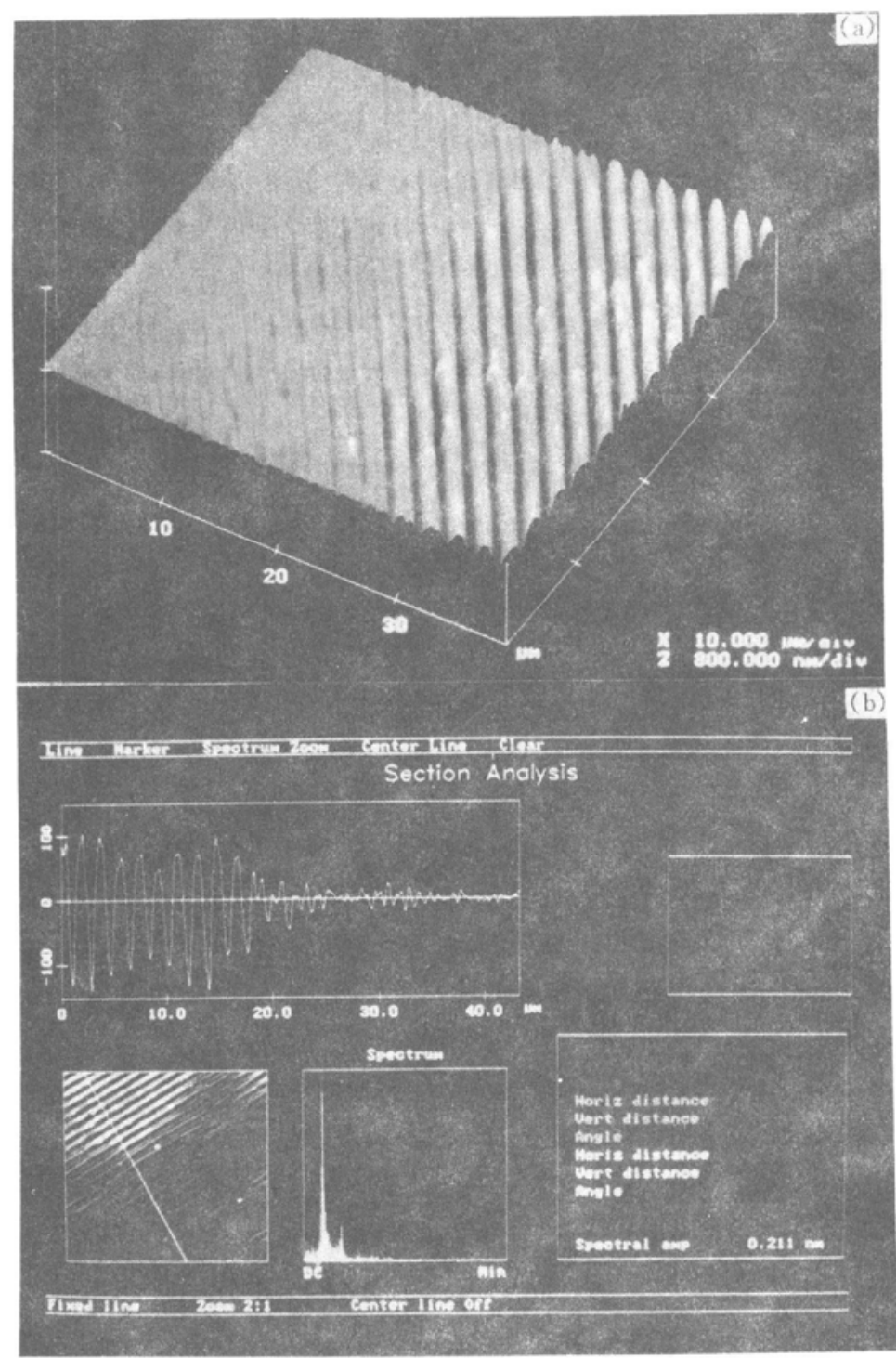

图 4 光相边界处的 AFM 形貌 (a) 三维结构; (b) 剖面结构 


\title{
【研究简报】
}

\section{多氯联苯 (PCBs) 分子的 $X$ 射线晶体学研究}

\author{
苗秀生 ${ }^{(1)}$ 储少岗 ${ }^{(1)}$ 徐晓白 ${ }^{(1 *}$ 金祥林 ${ }^{(2)}$ \\ (1)中国科学院生态环境研究中心, 北京 100085 ; (2) 北京大学物理化学研究所, 北京 $100083 . *$ 联系人)
}

\section{关铺词多县联萌体学 分子结构}

作为一类典型的环境污染物, 多氯联苯 (PCBs, polychlorinated biphenyls)早已引起人们的 广泛关注, 并对其进行了大量的研究工作, 这中间从色谱保持特征研究到理化参数的估算; 从 其在环境中的归趋与分布到生物代谢过程的研究; 从生物富集因子的计算到毒性作用机制的

出部分的面积与低于水平面部分的面积近似相等. 这一现象为本文提出的关于光柨形成的物 理过程提供了依据, 即光棚是在晶体表面的融化后再凝固过程中形成的.

当激光能量适中时, 发生在晶体表面的是一个激光熔融物理过程. 由于激光脉冲的时间很 短, 所以激光功率密度极高. 例如, 当一个能量为 $200 \mathrm{~mJ}$ 的激光脉冲 ( $30 \mathrm{~ns}$ ) 照射到面积为 0.25 $\mathrm{mm}^{2}$ 的晶体表面时, 它的功率密度约为 $50 \mathrm{MW} / \mathrm{cm}^{2}$, 如此高的功率密度可以立即将晶体表层融 化. 如果入射到晶体表面的脉冲激光能量在空间上呈周期分布, 则高能量区域的晶体在被融化 的同时, 由于激光脉冲的冲击作用, 向两边堆积, 从而形成表面的几何起伏. 从光棴的 AFM 照片 也可以看出,晶体表面的整体质量并没有减少, 只是重新分布, 并形成了几何光删.

由于激光脉冲的时间仅为几十个 ns, 所以融化的晶体表层会在空气中立即再结晶, 然后 相当于在空气中进行退火. 至于再凝固时光柵表面为何如此光滑, 可能与基片的单晶结构以 及样品表面的光洁度有关, 这个问题有待进一步的研究.

总之, 利用单个准分子激光脉冲和光楖模板, 本文在 LN 单晶薄片表面获得了具有光滑波 纹面的晶体光栅. 该技术在激光加工和制作集成光学器件 (如光䬻耦合器、光耚透镜等) 方面 有着重要的应用前景.

致谢 本工作为国家“禁登”计划、国家“八六三”计划、国家自然科学基金(批准号: 19425007) 和南京大学分析测试基金的部分资助项目。

\section{稄 考 文献}

1 Tamada $\mathrm{H}$, Yamada A. Saitoh $\mathrm{M}$. $\mathrm{LiNbO}_{3}$ thin-film optical wavcguidc grown by liquid phase epitaxy and its application to second-harmonic generation. J Appl Phys, 1991, 70: 2 536 2 541

2 Liu J M, Liu Z G, Wu Z C. Growth of $\mathrm{LiNbO}_{3}$ optical waveguide films by excimer laser ablation. Mater Lett, 1994, 20:35 $\sim 37$

3 Askins C G, Tsai T E, Williams G M, et al. Fiber-Bragg reflectors prepared by a single excimer pulse. Opt Lett, 1992, 17: $833 \sim 835$

4 Hill K O, Mola B, Bilodeau F, et al. Bragg gratings fabricated in monomode photosensitive optical fiber by UV exposure through a phase mask. Appl Phys Lett, 1993, 62: 1 035 1 037

(1997-02-18 收稿) 